\title{
Optical Rectification Effect on Chiral Metamaterials
}

\author{
Juliasih Partini ${ }^{1}$, Kamsul Abraha ${ }^{1}$, Arief Hermanto ${ }^{1}$, \\ Satoshi Tomita ${ }^{2}$, and Matsui Takahiro ${ }^{2}$ \\ ${ }^{1}$ Department of Physics, Universitas Gadjah Mada, Yogyakarta, Indonesia \\ ${ }^{2}$ Quantum Material Science Laboratory, Nara Institute of Science and Technology, Japan \\ e-mail : juliasih@ugm.ac.id
}

\begin{abstract}
Emission of terahertz (THz) radiation as a result of optical rectification of intense femtosecond laser pulses on chiral metamaterials has been observed. The chiral metamamaterials has simplified fabrication design but still capable to exhibit different refractive index for each circular polarization. Nonlinear response of metamaterials was observed by terahertz emission spectroscopy. The result plot clearly shows that the terahertz signal is sufficiently fit with a square of laser power. The phenomenon can be referred as optical rectification and can be exploited for the development of new THz sources.
\end{abstract}

\section{Keywords - Optical Rectification, Chiral Metamaterials, terahertz signal}

\section{INTRODUCTION}

Nanotechnology is the manipulation of matter on a nearatomic scale to produce new structures, materials and devices. This technology promises scientific advancement for many sectors such as medical, consumer products, energy, materials and manufacturing. Nanotechnology, in general terms covers engineered structures, devices, and length systems. At nanometers size, materials begin to exhibit unique properties that affect physical, chemical, and biological behavior. One of the results of nanotechnology is metamaterials.

Metamaterials are artificially structured materials that can be composed of dielectric elements or magnetic elements. The elemental structures of the metamaterials are typically much smaller in size relative to the wavelength. Metamaterials may possess electromagnetic properties that do not exist in natural material, such as negative refraction. Usually, negative refraction is obtained by adjusting the permittivity and permeability of the metamaterials to be negative simultaneously. Recently, it was proposed that a negative refraction can also be achieved alternatively through a chiral route [1]. A chiral material lacks any planes of mirror symmetry. In terms of electromagnetic responses, chiral metamaterials are characterized by a cross coupling between the electric and the magnetic dipoles along the same direction. With the strong chirality, negative refraction may occur for one circularly polarized wave, while for the other circular polarization the refraction index remains positive [2]. This gives rise to interesting phenomena that are not achieved by conventional metamaterials.

The nonlinear response of metamaterials is an essential and challenging task in the microwave to optical spectral range, especially in the terahertz $(\mathrm{THz})$ regime because of the lack of intense terahertz sources[3]. The recent development of a high-field terahertz source brings new opportunities to induce nonlinear effect, such as second harmonic generation, sum frequency generation, different frequency generation (DFG), parametric amplification and optical rectification. As we know, Rectification, producing electromagnetic radiation at terahertz frequencies from optical pulses, is a well-known phenomenon with a long history. However, due to the complexity of the chiral metamaterials geometry, experimental of chiral metamaterials at the terahertz and even higher frequencies still remain major challenges [4-9].

Here we study the generation of terahertz emission as a result of optical rectification of intense femtosecond laser pulses on chiral metamaterials using time domain $\mathrm{THz}$ spectroscopy. The result will be presented of terahertz pulse generation on the chiral metamaterials and the power dependence of terahertz-pulse generation. This research has been stimulated by the development of ultrafast lasers and of the emission and detection techniques that facilitated the introduction of time-domain terahertz spectroscopy.

\section{BACKGROUND}

Optical Rectification is widely used to rectify femtosecond laser pulses from the visible to the terahertz range. Although single cycle pulses based on optical rectification techniques have been available for almost three decades, only recently pulse energies exceeding one microjoule have become available on this systems. These sources are based on the process of optical rectification. The mechanism of terahertz pulse that can be viewed as the degenerate case of difference frequency generation for identical frequencies. Optical-rectification generates broadband $\mathrm{THz}$ pulses via difference frequency mixing of the frequency components of an ultra-short pulse. 
In a nonlinear optical material, the polarization induced by an AC electric field will not reverse its sign at the same time as the driving field, leading to a nonlinear polarization and thus frequency mixing. If the field is applied with a femtosecond laser pulse, with a large spectral bandwidth, frequency mixing of different frequency components results in electromagnetic waves in the terahertz region. This is the optical analog of the audible beat frequency generated by two struck tuning forks. The emitted field is related to the polarization. The generation of terahertz radiation via optical rectification is a second-order nonlinear optical effect. The process can be described by second or thirdorder nonlinear susceptibility tensor.

Optical rectification is a second-order nonlinear optics phenomenon with a special difference frequency. For a centrosymmetric crystal, the second order nonlinear susceptibility $\chi^{(2)}$ is equal to zero. Only the noncentrosymmetric nonlinear material can thus be selected to generate terahertz emission via optical rectification. The optical rectification is such a process that when an ultra short optical pulses incident on a nonlinear crystal, the input fields cause atoms to develop an oscillating dipole moment and radiate [10].

The nonlinear polarization can be written as

$$
P=P_{L}+P_{N L}=\varepsilon_{0} \chi^{(1)} \cdot E+\varepsilon_{0} \chi^{(2)} \cdot E E+\ldots
$$

We take the second order nonlinear polarization

$$
\begin{aligned}
P_{N L}^{(2)} & \propto \frac{1}{2} E_{0}^{2}(\cos 2 \omega t+1) \\
& \propto \frac{I_{0}}{2}(\cos 2 \omega t+1)
\end{aligned}
$$

There are two kind of optical rectification related to the virtual intermediate state. Those are coherent optical rectification and incoherent optical rectification. Coherent optical rectification is a second order nonlinear optical process in which the visible fields mix through the second order susceptibility tensor $\chi^{(2)}$ to produce a terahertz field [11]

$$
E_{T H z}(t) \propto \chi^{(2)} \frac{d I_{v i s}(t)}{d t},
$$

From the equation (3) we see that the terahertz field has a field shape approximately equal to the derivative of the envelope of the visible laser pulse. Coherent optical rectification is typically performed in noncentrosymmetric crystal such as ZnTe. In coherent optical rectification, the terahertz fluence depends on the square of the input power. In principle, optical rectification data can bring information about the second-order nonlinear susceptibility tensor that is complementary to that obtained by second harmonic generation, as optical rectification and second harmonic generation probe different spectral ranges. At the same time, the information is richer because of the phase sensitivity of time-domain spectroscopy. The time dependence of the radiated terahertz field can be analysed to reveal the dynamics of underlying nonlinear processes, as in the case of the terahertz emission spectroscopy that is widely applied in semiconductors for investigation of the ultrafast carrier dynamics.

In incoherent optical rectification, the visible laser pulse excites charge carriers to a more mobile state in which charge acceleration leads to the emission of radiation. It can be shown that the field of the emitted terahertz pulses in the far field is given by

$$
E_{T H z}(t) \propto \frac{d j}{d t} \quad,
$$

where $j$ is the surface current density. Molecular electron transfer involves the movement of charge within a molecule, which constitutes a microscopic current.

\section{SAMPLE PREPARATION \& EXPERIMENTAL SETUP}

The chiral metamaterials were fabricated by Focused Ion Beam System FB2200 in Graduate School of Materials Science, NAIST. There were 2 kind of sample with a period formed by a square pattern of chiral with different depth on a silver film. The first one is a clock-wise $(\mathrm{CW})$ sample (Fig. 1a) and the other is a counter clock-wise (CCW) sample (Fig. 1b). Figure 1 shows a single pattern chiral unit, with length $1.2 \mu \mathrm{m}$, width $0.3 \mu \mathrm{m}$ and depth $0.3 \mu \mathrm{m}$.
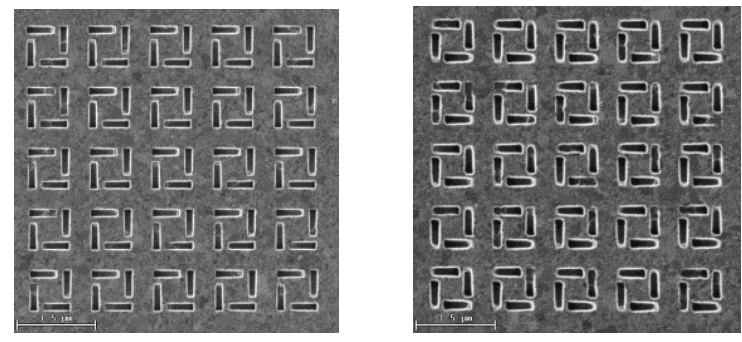

Figure 1. (a) Clock-wise (CW) sample (b) Counter clockwise $(\mathrm{CCW})$ sample.

The experiments were performed using a standard $\mathrm{THz}$ generation setup as shown in fig. 3. The laser source used is a Ti:Sapphire oscillator (Scientific XL, Femtolasers) that generates pulses of $50 \mathrm{fs}$ duration and centered at a wavelength of $800 \mathrm{~nm}$ with a repetition rate of $11 \mathrm{MHz}$. The average power output from the laser is $800 \mathrm{~mW}$. The $80 \%$ part of the is used as the pump beam and the $20 \%$ part as the sampling beam. The pump beam is weakly focused onto the sample surface to a spot size of about $2 \mathrm{~mm}$, at a $45^{\circ}$ angle of incidence. The $\mathrm{THz}$ radiation generated from the sample is collected in the specular reflection direction of the pump beam using off-axis paraboloidal mirror and focused onto a $500 \mu \mathrm{m}$ thick zinc telluride (ZnTe) detection crystal. The synchronized, coprpagating sampling pulse is also focused onto teh detection crystal. The $\mathrm{THz}$ electic field elliptically 
polarizes the probe beam to an extent proportional to the instantaneous $\mathrm{THz}$ electric field value. This ellipticity of the beam is measured by a differential detection setup consisting of a quarter wave plate, a Wollaston prism, and a differential detector.

An emitted $\mathrm{THz}$ electric field from the chiral metasurfaces is shown in Fig. 2. It consists of a nearly singlecycle followed by an oscillating tail. These weak oscillations are caused by the absorption and the re-emission of the $\mathrm{THz}$ light by the water vapor molecules in the atmosphere.

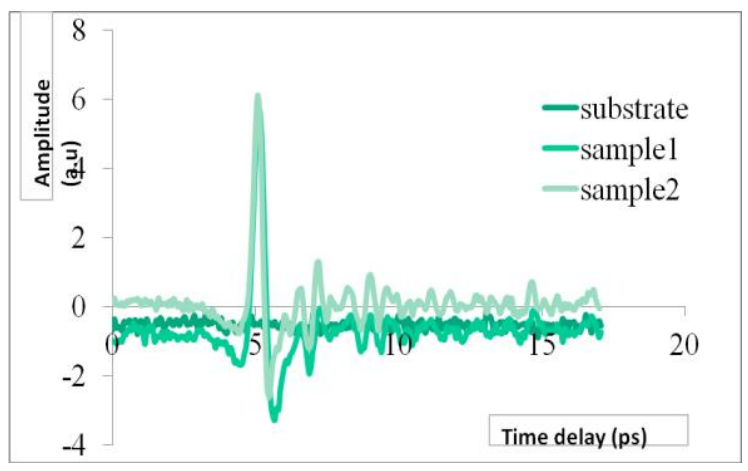

Figure 2. Spectra of the THz emission pulse.

We plot the measured $\mathrm{THz}$ amplitude as a function of incident laser power in Fig. 3. This experiments were performed to measure the terahertz fluence as a function of the 800-nm input power density. By varying the pump power, it is possible to obtain a power dependence of the terahertz emission from the sample. The terahertz fluence was calculated by fitting the data to an appropriate function. The measured input power dependence of the terahertz pulse fluence with least-squares fits to power laws. The sample data fits a power of 2 which is sufficiently consistent with the expected optical rectification process.

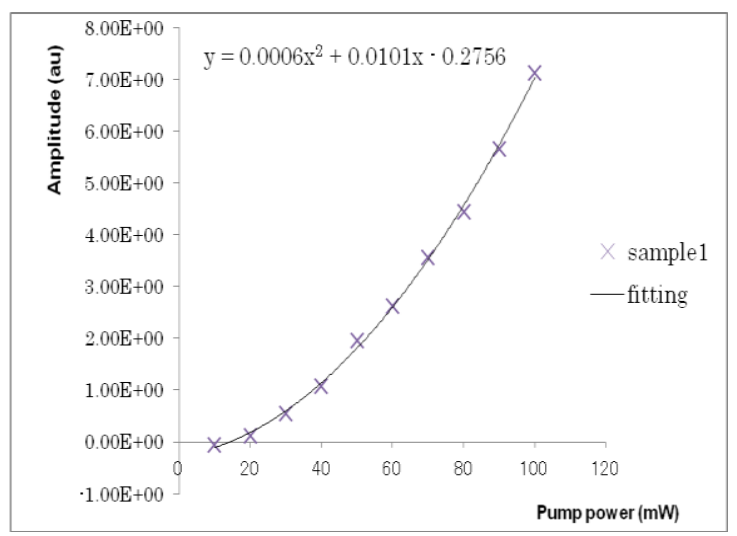

(a)

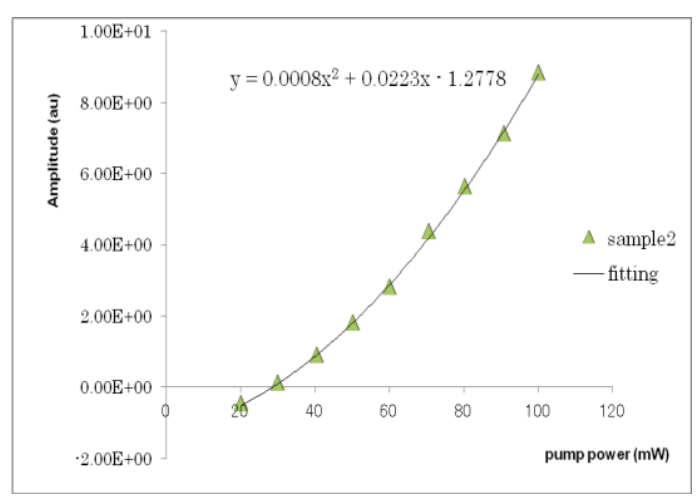

(b)

Figure 3. Input laser-power dependence of the terahertz pulse fluence in (a) sample 1 (b) sample 2.

\section{DISCUSSION}

It is clear that the optical rectification process responsible for terahertz emission in the chiral metamaterials is not just a simple second-order process. It is well known that metallic film irradiated with high power femtosecond laser pulses can emit photoelectrons in a process known as the nonlinear photoelectric effect. The nonlinear photoelectric effect is enhanced by surface roughness. It is interesting to note the second order dependence in these experiments. Optical rectification should result in a second-order dependence on input fluence as reported by Liu et al[12]. The presence of a surface breaks the translational symmetry, thereby allowing $\chi^{(2)}$ processes to take place with the sample. It is well known that this accounts for second-harmonic generation on surfaces and could well explain optical rectification on surface too.

$\mathrm{THz}$ amplitude clearly increases non linearly, suggesting that a second-order non linear process is responsible for the $\mathrm{THz}$ emission. This consistent with the earlier observation by Kadlec et all, where a higher order non linear process resulted the $\mathrm{THz}$ emission from an Au surface [13]. On the other hand, they reported a second order process from $\mathrm{Au}$ films only above a thickness of $100 \mathrm{~nm}$. A direct comparison of their work with our work is difficult, however, as percolated metal film are known to behave differently for metal. But at least, we have the same process that that responsible for the $\mathrm{THz}$ emission. Enhanced of second order nonlinear processes like SHG has been reported from percolated Ag film on glass [12]. Lack of inversion symmetry is essential for such a process. For a ultrathin Ag flim, the inversion symmetry is broken in the 
direction perpendicular to the sample surface. Femtosecond laser excitation can thus lead to the formation of a $\mathrm{THz}$ dipole oriented normal to the sample surface, through a second order optical rectification process. This is suggest that the degree of on linear process plays a role in the $\mathrm{THz}$ emission process.

\section{CONCLUSION}

From the description of $\mathrm{THz}$ emission of chiral metasurface due to the optical rectification processes, we may conclude the generation of $\mathrm{THz}$ emission pulses of chiral meta-surface with femtosecond laser pulses. These second order power dependence of the terahertz pulse fluence observations which is sufficiently consistent with the expected optical rectification process.

\section{ACKNOWLEDGMENT}

We gratefully acknowledge Matsui Takahiro from NAIST Japan for fabricating samples and the team of Laser Terahertz Division in Osaka University for the terahertz emission experiments.

\section{REFERENCES}

[1] J. B. Pendry, "A Chiral Route to Negative Refraction", Science, vol. 306,pp. 1353, November 2004.

[2] S. Zhang, Y.-S. Park, J. Li, X. Lu, W. Zhang and X. Zhang, "Negative Refractive Index in Chiral Metamaterials", Phys. Rev. Lett., vol. 102, pp. 023901, January 2009.

[3] A. A. Zharov, I.V. Shadrivov and Y. V. Kivshar, "Nonlinear properties of left-handed mematerials", Phys. Rev. Lett. Vol. 91, 2003.

[4] Gopakumar Ramakrishnan and Paul C. M. Planken, "Percolation enhanced generation of terahertz pulses by optical rectification on ultrathin gold films", Opt. Lett., vol. 36 no. 13, pp. 2572-2574, 2011.

[5] N. Kanda, K. Konishiand, and M. Kuwata-Gonokami, "Terahertz wave polarization rotation with double layered metal gratting of complimentary chiral patterns", Opt. Exp. 11117, vol 15 No. 18, 2007.

[6] M. Kuwata-Gonokami, "Giant Optical Activity in Quasi-TwoDimensional Planar Nanostructures", Phys. Rev. Lett. Vol. 95, pp. 227401, 2005.

[7] T. Hatano, B. Nishikawa, M. Iwanaga and T. Ishihara, "Optical rectification effect in 1D metallic photonic crystal slabs with asymmetric unit cell", Opt. Exp., vol. 16 no. 11, pp. 8236, 2008.

[8] F. Kadlec, P. Kuzel and J. L. Countaz, "Study of terahertz radiation generated by optical rectification on thin gold films", Opt. Lett., vol. 30, No. 11, pp. 1402-1404, 2005.

[9] J. Nishitani, K. Kozuki, T. Nagashima and M. Hangyo, "Terahertz radiation from coherent antiferromagnetic magnons excited by femtosecond laser pulses", App. Phys. Lett. Vol. 96, pp. 221906, 2010.

[10] L. Dehua, Q. X. Dong and L. S. Gang, "A theoretical analysis of optical-to-THz conversion efficiency via optical rectification", Sci. China Ser E-Tech .Sci, vol. 51 no. 12, pp. 2080-2088, July 2008.

[11] G. H. Welsh dan K. Wynne, "Generation of ultrafast terahertz radiation pulses on metallic nanostructured surfaces", Opt. Exp., vol. 17, No. 4, pp. 2470-2480, 2009.
[12] H. Liu, G. X. Li, K. F. Li, S. M. Chen, S. N. Zhu, C. T. Chan and K. W. Cheah, "Linear and nonlinear Fano resonance on two dimensional magnetic metamaterials", Phys. Rev. B, vol. 84, pp. 235437, 2011.

[13] F. Kadlec., Kuzel., and J. L. Coutaz," Study of terahertz radiation generate by optical rectification on thin gold film", Opt. Lett, vol. 30, pp. 1402, 2005. 